\title{
A New Method for Controlling and Maintaining Topology in Wireless Sensor Networks
}

\author{
Aminuddin $\mathrm{Zabi}^{1}$, Touseef Yousuf ${ }^{2}$ and Aparna Manikonda ${ }^{3}$ \\ Department of Computer Science Engineering \\ Nitte Meenakshi Institute of Technology, Bengaluru-64, India
}

\begin{abstract}
Topology Control is an essential technique in a wireless sensor network to extend the operational time of the sensor nodes. The goal of this technique is to maintain network connectivity and optimize performance metrics such as network lifetime and throughput. In this paper we presented a new method for controlling and maintaining topology in wireless sensor networks that show some improvement over the state of art methods. The results are analyzed based on objective criteria.
\end{abstract}

\section{KEYWORDS}

Wireless Sensor Networks, ENR, Topology Control, Topology Construction, Topology Maintenance.

\section{INTRODUCTION}

A wireless sensor network is a reliant collection of nodes that communicate over relatively bandwidth constrained wireless links. In general, topology control in sensor networks adjusts their transmission ranges to conserve energy and reduce interference. In case of wireless sensor networks the aspects which need to be considered while controlling topology are operation in hostile environments, Data processing and scalability. A significant amount of research works [14] have been done using non homogenous approaches such as location based, neighbor based and direction based.

In this paper we devised a new method called ENR which stands for Energy-Neighbour-Range to control and maintain topology in wireless sensor networks. Our approach is fully distributed, asynchronous and generates a connected topology. The method is based on the principle of maintaining the number of physical neighbours, transmission range and energy levels at each node while constructing topology in order to improve the efficiency of the network.

The rest of the paper is organized as follows. Section 2 briefly describes the taxonomy of topology control methods. Section 3 presents our working principle. Section 4 presents the simulation environment and experimental results. Finally conclusions are drawn in Section 5.

\section{TAXONOMY OF TOPOLOGY CONTROL}

Topology control is an essential technique in Wireless sensor network which leads to reorganizing and managing of certain node parameters and modes of operation from time to time to modify the topology of the network with the goal of extending its lifetime while preserving important characteristics such as connectivity and coverage. Topology control is an iterative process which consists of two phases those are topology construction and topology maintenance. 
During the topology construction phase, each node builds a new topology based on the best parent node. Nodes can extend the network lifetime by adjusting transmission power to control the topology. In the topology maintenance phase, each node monitors the energy status of neighbours and triggers topology construction as needed. The framework of topology control approaches as shown in figure below:

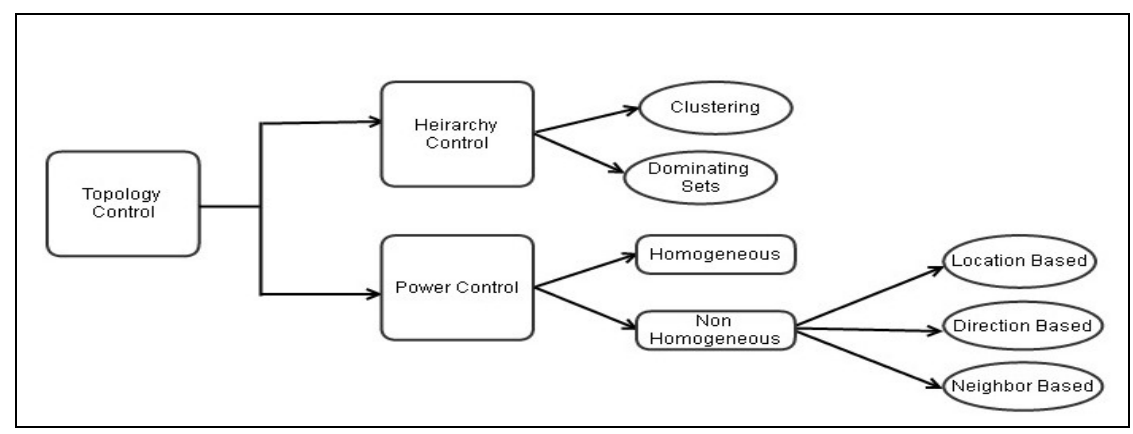

Fig. 1. Taxonomy of Topology Control[5]

In homogeneous topology control, the transmitting range of the nodes is the critical transmitting range that produces communicating graphs that are connected with high probability. Determining the critical transmission range using homogeneous topology control has been considered analytically as well as practically.

In Nonhomogeneous approach the transmitting range of the nodes varies and is classified into three categories, depending on the type of information that is used to compute the topology. In location based approaches, exact node positions are known. In direction-based approaches, it is assumed that nodes do not know their position, but they can estimate the relative direction of each of their neighbors. Finally, in neighbor-based techniques, nodes are assumed to know only the ID of the neighbors and are able to order them according to some criterion.

\section{PROPOSED SCHEME}

In this section we briefly describe the framework of ENR algorithm and its operating phases i.e. (1) topology construction phase and (2) topology maintenance phase.

\subsection{Frame Work}

The frame work of the ENR algorithm is described in the following steps as follows and as shown below in the figure 2 :

Step-1: Initially, we get the energy of the randomly deployed nodes by using node energy model [1] that can accurately reveal the energy associated with each node.

Step-2: Next we will be determining the neighbors [2] of the sensor nodes; our method relies on distance estimation and the energy of the node.

Step-3: The maintenance of the proposed system is ensured by selecting the other node from the neighbor data structure [4] in case of energy depletion.

Step-4: Maintenance process involves periodic restoration and recreation of network topology. Here the each node periodically broadcasts the hello messages to its neighbor and waits for the acknowledgement. 


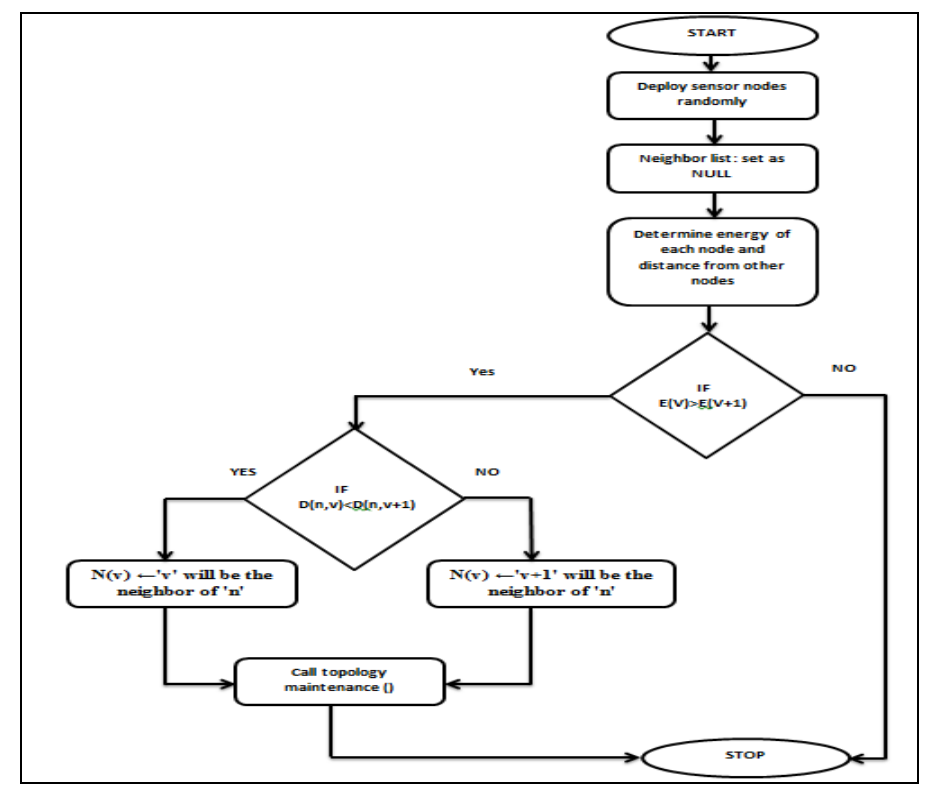

\subsection{Topology Construction}

Fig. 2. Frame Work of ENR Algorithm.

In topology construction phase, an efficient methodology is used that constructs a topology dynamically without violating network scenarios and thereby providing both connectivity and coverage. Hence a topology construction algorithm is proposed for building a topology and the steps for the same as shown in the figure 3.

We start constructing the topology $T$ (v) where each node ' $n$ ' selects its neighbor ' $v$ ' based on range, energy and distance. Initially the neighbor list of a node is set to Null (line 2). In order to select the neighbor we determine the energy of each node $\mathrm{E}(\mathrm{v})($ line 3-4). Now the node list $\mathrm{E}(\mathrm{v})$ is sorted in decreasing order(Line 6).After computing the energy we find the distance of each node ' $v$ ' from ' $n$ ' in order to determine the neighbor list of every node (Line 10-14).The nodes which are not present in the neighbor list $\mathrm{N}(\mathrm{v})$ is set to inactive state.

\subsection{Topology Maintenance}

In topology maintenance phase, as node start communicating with each other the energy depletes and some nodes fail rendering the network useless. Hence to make network functioning optimal we need to provide topology maintenance [4]. This process involves periodic restoration, rotation and recreation of network topology. Here the node periodically broadcasts the hello messages to its neighbor and waits for the acknowledgement(line 1-2).The Hello message carries the following information:<node_id,neighbor_id,energy>. If the node does not receive back acknowledgment (if the neighboring node is not having the energy to broadcast a message) then it invokes topology construction function. the steps as shown in figure 4. 


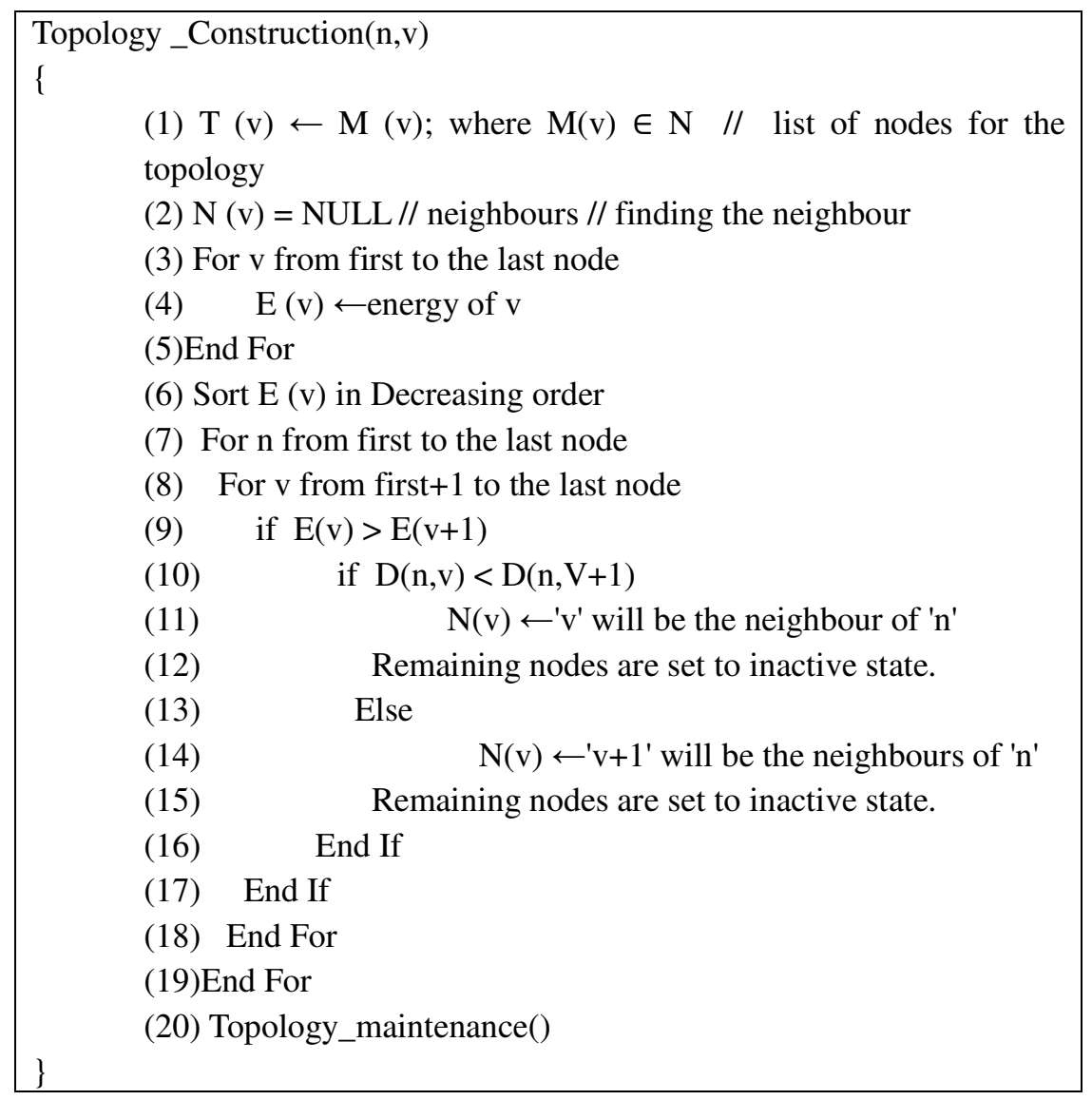

Fig. 3. Topology Construction Algorithm.

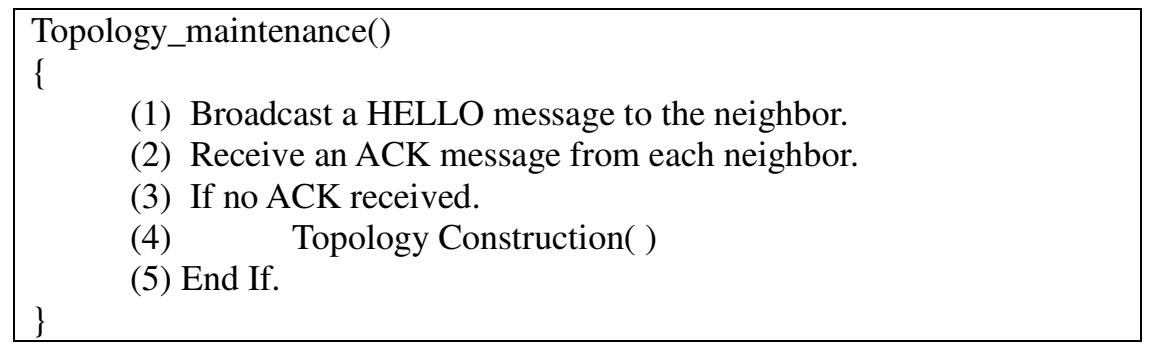

Fig. 4. Topology Maintenance Algorithm.

\section{Simulation Environment}

\subsection{Simulation model and parameters}

We use ns2.33[7] to simulate our proposed protocol. In our simulation, we use the DCF of IEEE 802.11 for wireless LANs as the MAC layer protocol. It has the functionality to notify the network layer about link breakage.

Our simulation settings and parameters are summarized in table below: 
International Journal of Computer Networks \& Communications (IJCNC) Vol.6, No.4, July 2014

\begin{tabular}{|l|l|}
\hline \multicolumn{1}{|c|}{ Parameter } & \multicolumn{1}{|c|}{ Value } \\
\hline Number of simulated Nodes & 70 \\
\hline Pause time & $10 \mathrm{sec}$ \\
\hline Area size of topography (m) & $1600 * 2550 \mathrm{~m}$ \\
\hline Radio range & $250 \mathrm{~m}$ \\
\hline Packet size & 512 bytes \\
\hline Send rate of traffic & 10 packets/sec \\
\hline Traffic type & TCP \\
\hline MAC Type & 802.11 \\
\hline Simulation Time & $150 \mathrm{~s}$ \\
\hline Simulated Routing Protocols & ENR,CBTC,K-Neighbour \\
\hline
\end{tabular}

\subsection{Performance Metrics}

Table-1: Simulation Parameters.

We evaluate mainly the performance of ENR algorithm according to the following metrics, by varying the pause time.

Throughput: It is the number of bits that are conveyed or processed per unit of time.

Packet Delivery Ratio: It is the ratio of the no. of packets received successfully and the total no. of packets sent.

Bit Error Rate: It is the percentage of bits with errors divided by the total no. of bits transmitter, received or processed over a given time period.

\subsection{Results}

In this experiment, we measure the performance of the protocols at different pause time.

In Fig 5, Initially throughput increases with the increase in time. Initial increase in throughput is due to multiple route capability of ENR protocol (when the time increases from 0 to $5 \mathrm{sec}$ ). However as the time further increases the throughput decreases due to the dropping of packets due to the network congestion (this is shown when time increases from 5 to 50). Further from above plot we can determine our ENR protocol provides better throughput than CBTC and KNeighbor protocol and maximum throughput is achieved when time is $5 \mathrm{sec}$. 
International Journal of Computer Networks \& Communications (IJCNC) Vol.6, No.4, July 2014

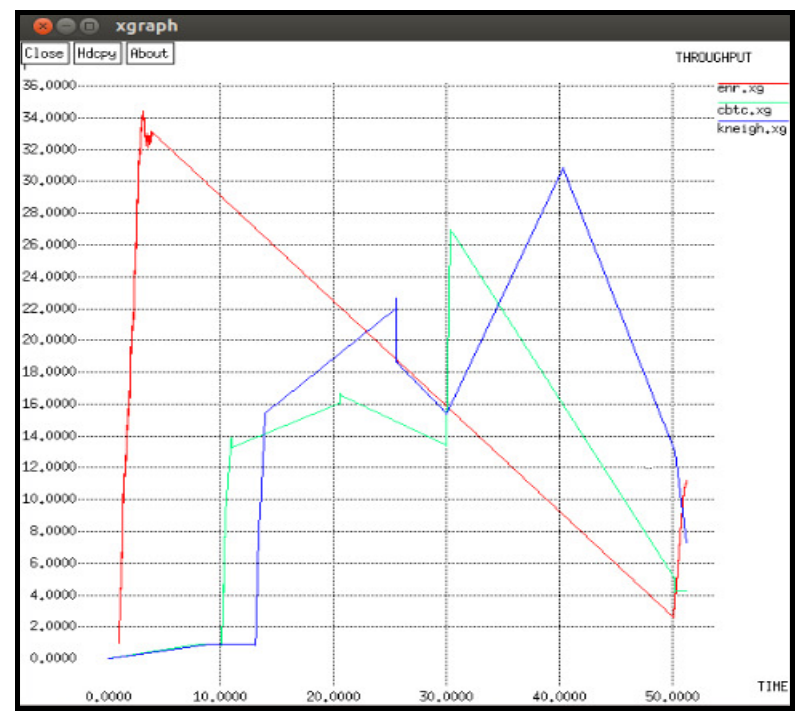

Fig. 5. Throughput vs. pause time.

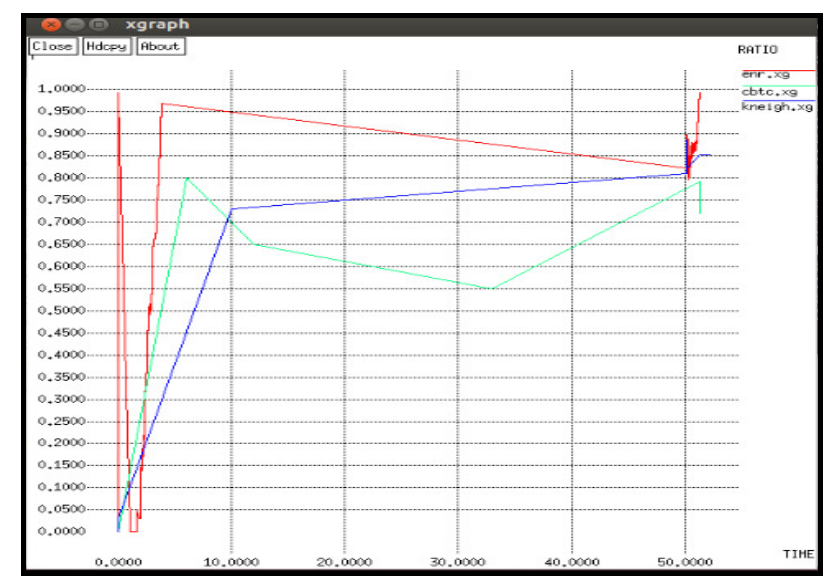

Fig. 6. Packet Delivery ratio vs. pause time.

In fig 6, as the time increase the number of possible paths between source and sink pair increases which causes a significant increase in the exploratory messages within in network which initially leads to higher packet delivery ratio of about (95-100\% in case of ENR). With further increase in the time, the performance of packet delivery ratio decreases due to congestion which in turn leads to collision. However in case of ENR protocol packet delivery ratio remains somewhat stabilized as compared with CBTC and K-Neighbor. 
International Journal of Computer Networks \& Communications (IJCNC) Vol.6, No.4, July 2014

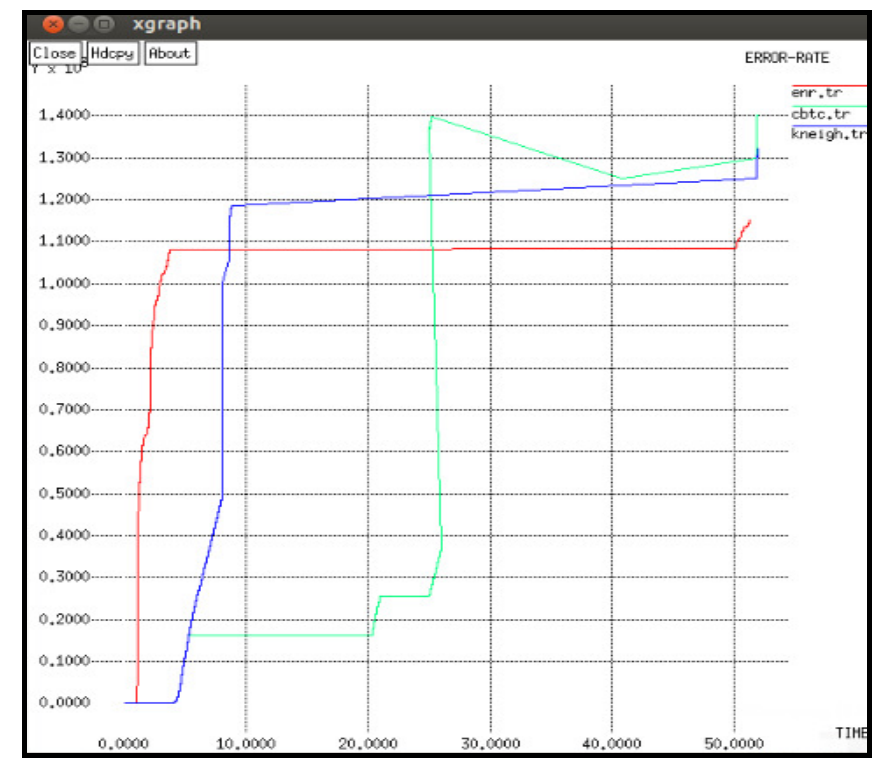

Fig. 7. Bit Error Rate vs. pause time.

In fig 7, initially when we are sending data the percentage of error rate is high due to noise or interference. However as the time increases the error rate of ENR is comparatively less as compared with CBTC and K-neighbor. As Error rate decreases in ENR the throughput increases, the error rate is decreased with the help of varying the FEC code size so that the erroneous packets are corrected instead of going for retransmission which improves the throughput.

\section{Conclusion}

In this paper we have proposed and implemented ENR (energy neighbour range) algorithm. This algorithm performs better in variable sized networks as compared with the the state of art methods. Our algorithm adopts a non-homogeneous approach which takes into consideration location, direction and neighbor information of a system in a network. However our algorithm results in some overhead as it needs to compute various parameters like energy, range and neighbors. This is overshadowed by high coverage and enhancing the lifetime of a network.

\section{REFERENCES}

[1] Hai-Ying Zhou, Dan-Yan Luo, Yan Gao, De-Cheng Zuo, (2011) "Modeling of Node Energy Consumption for Wireless Sensor Networks", Wireless Sensor Network, Vol 3, pp. 18-23.

[2] Runze Ma, Zhijun Yu , Haitao Liu (2012)" The Neighbors Selection Algorithm in WSN for Routing and Localization" Journal of Information \& Computational Science Vol.9, No.4, pp. 1017 -1025 .

[3] Abderrahim Tahiri, Esteban Egea-López, Javier Vales-Alonso, Joan García-Haro \& Mohamed Essaaidi(2009) “A Novel Approach For Optimal Wireless Sensor Network Deployment", Symposium on Progress in Information \& Communication Technology 2009.

[4] A.Karthikeyan, T.Shankar, V.Srividhya, Siva Charan Reddy.v, Sandeep Kommineni(2013), "Topology Control Algorithm For Better Sensing Coverage With Connectivity In Wireless Sensor. Networks", Journal of Theoretical and Applied Information Technology., Vol.52, No.3,pp.308 
International Journal of Computer Networks \& Communications (IJCNC) Vol.6, No.4, July 2014

[5] Muhammad Asghar Khan, Asfandyar Khan, Said Khalid Shah, Azween Abdullah,(2013) “An Energy Efficient Color Based Topology Control Algorithm for Wireless Sensor Networks" Wireless Sensor Network, Vol. 5,No. 1, pp.1-7.

[6] Paolo Santi(2005) "Topology Control in Wireless Ad Hoc and Sensor Networks", ACM Computing Surveys, Vol. 37, No. 2, pp. 164-194.

[7] The Network Simulator, NS-2.34, Available from www.isi.edu/nsnam/ns, 2009

\section{AUTHORS}

Aminuddin Zabi received his BE degree from Visvesvaraya Technological University in 2012 and he is currently pursuing his master's degree (M.TECH) at Nitte Meenakshi Institute of technology, Bangalore, affiliated to Visvesvaraya Technological University, India. His research interests include the study of structural properties and information security in wireless sensor networks.

Touseef Yousuf received his BE degree from University of Kashmir and he is currently pursuing his master's degree (M.TECH) in computer science and engineering at Nitte Meenakshi Institute of technology, Bangalore, affiliated to Visvesvaraya Technological University, India. His research interests include wireless sensor networks, cloud computing and Algorithm design.

Aparna Manikonda received her BE and MTECH degree from Biju patnaik university of technology. She is currently working as an assistant professor in the department of computer science and engineering at Nitte Meenakshi Institute of technology, Bangalore, affiliated to Visvesvaraya Technological University, India. Her research interests include Adhoc and Sensor networks, Data Mining and Warehousing, Cloud computing and Image processing.
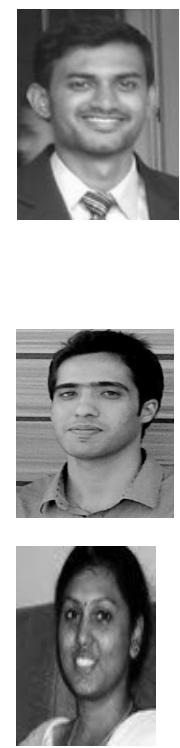